\title{
Microwave-Sustained Miniature Plasmas for an Ultra Small Thruster *
}

\author{
Yoshinori Takao *,1, Kouichi Ono, Kazuo Takahashi, \\ Yuichi Setsuhara ${ }^{2}$ \\ Department of Aeronautics and Astronautics, Graduate School of Engineering, \\ Kyoto University, Yoshida-Honmachi, Sakyo-ku, Kyoto 606-8501, Japan
}

\begin{abstract}
This paper proposes an application of microwave-sustained miniature plasmas to an ultra small thruster for next-generation microspacecraft, and presents some results of numerical investigations of its thrust performance and preliminary experiments of Ar plasma discharges. Numerical results show that the thruster has enough thrust performance for a station-keeping maneuver of microspacecraft below the power of $10 \mathrm{~W}$. Optical emission spectrometry implies a mode change in the plasma discharges inside the quartz tube $1.5 \mathrm{~mm}$ in inner diameter using 4-GHz microwaves at powers between $6 \mathrm{~W}$ and $10 \mathrm{~W}$.
\end{abstract}

Key words: Computer simulation, Optical spectroscopy, Microplasma, Microthruster 


\section{Introduction}

In recent years, developing microplasma sources has attracted increasing attentions, aimed at applications in microsystems. Such microplasma sources are obtained by dc plasma [1], capacitively coupled plasma [2], inductively coupled plasma [3], and microwave-excited plasma [4]. For space systems, there is an ongoing trend toward miniaturization and simplification of spacecraft to reduce mission costs and risk [5]. Such microspacecraft concept has supported a new approach to develop accurate, reliable, and low-power-consumption micropropulsion systems for high-precision station keeping (requiring $\sim \mathrm{mN}$ thrust) and attitude control $(\sim \mu \mathrm{N})$.

In this work, we present an application of a miniature plasma source excited by microwaves to an ultra small thruster on microspacecraft. Figure 1 shows a schematic diagram of the microplasma thruster, which consists of a microplasma source and a micronozzle. The plasma source is composed of a cylindrical dielectric tube, the outside of which is covered with a metal grounded. The inner radius and the length of the tube are $1 \mathrm{~mm}$ and $10 \mathrm{~mm}$, respectively. Microwaves are injected through a coaxial cable into the plasma chamber, where propellant, argon for this study, is ionized and heated up

‡ Work supported by a Grant-in-Aid for Scientific Research from Ministry of Education, Culture, Sports and Science and Technology, Japan.

* Corresponding author Email address: yoshinori_takao@kuaero.kyoto-u.ac.jp (Yoshinori Takao).

1 Supported by Research Fellowships of the Japan Society for the Promotion of Science for Young Scientists.

2 Present Address: Joining and Welding Research Institute, Osaka University, 11-1, Mihogaoka, Ibaraki, Osaka 567-0047, Japan 
in the pressure range from $10 \mathrm{kPa}$ to $100 \mathrm{kPa}$. Such high thermal energy is converted into the kinetic energy through the micronozzle, which has a converging and diverging section, and then the thrust is obtained. On the basis of this concept, a numerical model has been developed to investigate the thrust performance. Then, as a preliminary experiment, we have fabricated only a microplasma source, observing plasma discharges and their emission spectra. Some results are shown in the following sections.

\section{$2 \quad$ Numerical investigations}

In this section, the main purpose is to estimate the thrust performance using simple models without time-consuming calculations. Since the plasma obviously has different characteristics in the reservoir or nozzle, separate models have been developed. The assumption of the models is as follows: the plasma is a continuum, singly ionized, quasi-neutral, and a two-phase medium that is composed of heavy particles (atoms and ions) and electrons with different temperatures; the chamber has no flow, volume-averaged plasma parameters such as electron density, and axisymmetric electromagnetic structure, while the nozzle has laminar and axisymmetric plasma flows. From these assumptions, we employ a global model module [6], and an electromagnetic model module using the finite-difference time-domain (FDTD) method for the plasma source $[7,8]$, while a fluid model module based on two-temperature NavierStokes equations with the finite difference method for the nozzle flow [9]. The model equations are described in Ref. [10].

Figure 2 (a) shows the dependence of plasma parameters on the absorbed power in plasmas $P_{\mathrm{abs}}$ at the pressure of $100 \mathrm{kPa}$, the microwave frequency 
$f$ of $4 \mathrm{GHz}$, and the dielectric constant $\varepsilon_{\mathrm{d}}$ of 3.8 . The electron density $n_{\mathrm{e}}$, the electron temperature $T_{\mathrm{e}}$, and the heavy particle temperature $T_{\mathrm{h}}$ have been calculated as $1.5 \times 10^{20}$ to $1.7 \times 10^{22} \mathrm{~m}^{-3}, 8.2 \times 10^{3}$ to $1.0 \times 10^{4} \mathrm{~K}$, and $1.8 \times 10^{3}$ to $1.0 \times 10^{4} \mathrm{~K}$, respectively, in the absorbed power range from 1 to $10 \mathrm{~W}$. The electron density increases almost linearly with increasing $P_{\text {abs }}$; this result leads to the increase in $T_{\mathrm{h}}$ because of a high rate of the energy exchange through the elastic collisions between electrons and heavy particles. The figure indicates that $T_{\mathrm{h}}$ almost equals $T_{\mathrm{e}}$ above $P_{\mathrm{abs}}=7 \mathrm{~W}$, where even in a small space, enough electron density for the thermal equilibrium is obtained owing to a high power density on the order of $10^{8} \mathrm{~W} / \mathrm{m}^{3}$. When $n_{\mathrm{e}}$ increases, however, the radiative energy loss, which is also included in the model, becomes significant, suppressing $T_{\mathrm{e}}$. Moreover, the elastic energy exchange rate is proportional to the difference between $T_{\mathrm{e}}$ and $T_{\mathrm{h}}$. As a result, the increasing rate of $T_{\mathrm{h}}$ decreases with increasing $P_{\text {abs }}$.

The FDTD analysis indicates that $P_{\text {abs }}$ tends to increase with increasing $f$ and $\varepsilon_{\mathrm{d}}$. However, we have found that under some certain combinations between them, coupling microwaves to plasmas is rather effective, where a kind of cavity resonance would be expected. The validity of this result is confirmed using the theoretical dispersion equation. Details are discussed in Ref. [10].

Next, in order to estimate the thrust performance of the thruster, nozzleflow calculations have been performed using the results shown in Fig. 2 (a) as the inlet conditions, and the dependence of the thrust and the boundary layer thickness on $P_{\text {abs }}$ is demonstrated in Fig. 2 (b). The thickness of the boundary layer $\delta$ is defined as $\delta=5 / R e^{1 / 2}$, where $R e$ is the Reynolds number calculated at the nozzle throat [11]. Although the thrust increases with $P_{\text {abs }}$, the rate of increase tends to decrease, because of the increase in $\delta$ near the 
nozzle wall, where viscous dissipation occurs, as well as of the decrease in the increasing rate of the heavy particle temperature. This result indicates that providing as much power as possible does not directly improve the thrust performance. Nonetheless, the thruster yields a few $\mathrm{mN}$ levels of the thrust in the given power range, which means that the thruster could be used for a station-keeping maneuver of microspacecraft.

\section{$3 \quad$ Experimental setup}

A schematic diagram of the experimental setup and a cross-section view of the microplasma source are shown in Fig. 3. A commercially available Synthesized RF Signal Generator, $9 \mathrm{kHz}$ - $4000 \mathrm{MHz}$ (Agilent Technologies, 8648D) is employed as a 4-GHz microwave oscillator. Microwaves, amplified with a specially fabricated amplifier (Micro Denshi, MRF-4G-2), are injected through a semi-rigid type coaxial cable into the plasma source made of a straight quartz tube. The end of the cable is covered with quartz to protect the electrodes from erosion by plasmas. The quartz tube, connected to the stainless-steel pipe through which the working medium, Ar is introduced, is inserted into a stainless-steal chamber with $40 \mathrm{~cm}$ in diameter and $40 \mathrm{~cm}$ in height, which is evacuated by a mechanical booster dry pump. The size of the tube is $10 \mathrm{~mm}$ in length and $1.5 \mathrm{~mm}$ in inner diameter; the end of the tube is pinched and its diameter is reduced to about $0.8 \mathrm{~mm}$. This structure can make the conductance small to keep the pressure inside the quartz tube much higher than that in the vacuum chamber without a micronozzle. The outside of the quartz tube is covered with metallic mesh instead of metallic pipe for the observation of Ar plasma emission. The emission intensities are measured with a spectrom- 
eter (Ocean Optics, HR2000CG-UV-NIR) through an optical fiber, the head of which is directly positioned in front of the window of the chamber without a lens. A pressure gauge, equipped with the stainless-steal pipe between the mass flow controller and the plasma source, is used for the measurement of the feed pressure, which is assumed to be the pressure inside the quartz tube where plasmas are generated. Another gauge with the vacuum chamber measures the ambient pressure of the quartz tube.

\section{Experimental results and discussion}

Figure 4 shows the emission spectra and photographs of Ar plasma discharges at the incident power from the amplifier $P_{\text {in }}$ of $6 \mathrm{~W}$ (a) and $10 \mathrm{~W}(\mathrm{~b})$, where the microwave frequency was $4 \mathrm{GHz}$, the argon flow rate was $100 \mathrm{sccm}$, the feed pressure was $10 \mathrm{kPa}$, and the ambient pressure was $16 \mathrm{~Pa}$. Note that the power $P_{\text {in }}$ is not the net absorbed power in plasmas, but the incident power from the amplifier. The plasma was not found to self-ignite in the current setup, and therefore the plasma was generated with the help of an igniter. As shown in the figure, the plasma was maintained only inside the quartz tube at $P_{\text {in }}=6 \mathrm{~W}$, while the plasma was blown out to the chamber as a free jet at $P_{\text {in }}=10 \mathrm{~W}$. The color of the plasma was dim and purplish red at $6 \mathrm{~W}$, whereas it became bright and whitish pink at $10 \mathrm{~W}$; the emission intensities show this difference. When we focus on the strongest emission line, $763.5 \mathrm{~nm}$ $\left(1 \mathrm{~s}_{5}-2 \mathrm{p}_{6}\right.$ transition), and the second strongest, $811.5 \mathrm{~nm}\left(1 \mathrm{~s}_{5}-2 \mathrm{p}_{9}\right.$ transition), the ratio of the two spectra intensities $I$ differs slightly $\left(I_{763.5} / I_{811.5}=1.47\right.$ at $P_{\text {in }}=6 \mathrm{~W}, I_{763.5} / I_{811.5}=1.13$ at $\left.P_{\text {in }}=10 \mathrm{~W}\right)$, reflecting the change in the state of the plasma. This change suddenly occurred as $P_{\text {in }}$ increased. Once the 
shift happened, the increase in emission intensities was imperceptible despite the power rise.

Figure 5 shows the dependence of emission intensities of Ar I line $(811.5 \mathrm{~nm})$ (a) and of reflective coefficients (b) on $P_{\text {in }}$, where the experimental conditions were the same as those in Fig. 4, and the reflective coefficient was defined as the ratio of the reflective power to the incident power. The figure shows that, with increase in $P_{\mathrm{in}}$, the Ar I line intensity increases slightly, rises sharply at $P_{\text {in }}=9 \mathrm{~W}$, and then increases gradually again. As $P_{\text {in }}$ decreases, the intensity decreases and then drops steeply at $P_{\text {in }}=6.5 \mathrm{~W}$. The reflective coefficient drops or rises drastically at the same power where the significant change of intensity occurs. These results were found to be reproducible, implying that a mode change occurred at incident powers between $6 \mathrm{~W}$ and $10 \mathrm{~W}$.

The mechanism of this intensity transition would be due to the difference of the way microwaves propagate in the plasma: in the low-intensity mode, microwaves were attenuated upon entering the plasma chamber from the end of the coaxial cable, while in the high-intensity mode, propagated along the plasma-dielectric interface, so that the plasma was sustained even outside in the vicinity of the chamber exit. Since higher electron densities are expected at higher intensities and larger microwaves power, we ascribe this difference to whether or not the electron density exceeds the surface-wave resonance density $n_{\mathrm{SW}}$, where $n_{\mathrm{SW}}=9.5 \times 10^{17} \mathrm{~m}^{-3}$ at $f=4 \mathrm{GHz}$ and $\varepsilon_{\mathrm{d}}=3.8$. To confirm this, further optical and electrical diagnostics are underway.

Finally, to investigate the effect of flow on plasma discharges, experiments have been conducted for various flow rates. Figure 6 shows the dependence of emission intensities of Ar I line $(811.5 \mathrm{~nm})$ on $P_{\text {in }}$ for three argon flow rates: 
$100 \mathrm{sccm} ; 200 \mathrm{sccm}$; and $300 \mathrm{sccm}$, where corresponding feed pressures and ambient pressures were $10 \mathrm{kPa}, 16 \mathrm{~Pa} ; 15 \mathrm{kPa}, 23 \mathrm{~Pa}$; and $20 \mathrm{kPa}, 29 \mathrm{~Pa}$, respectively, and the microwave frequency was $4 \mathrm{GHz}$. Notice that the values of reflective coefficient at the flow rates of 200 and $300 \mathrm{sccm}$ were between 0.25 and 0.35 , showing little difference from those in Fig. 5 (b). As shown in the figure, the power range where the hysteresis occurs has a tendency to become smaller with increasing flow rate, whereas at lower or higher powers the power dependence of intensities appears to become larger as the flow rate increases. These results indicate that the flow had an effect on plasma discharges.

In view of the fact that the power supply and the propellant storage are strictly limited for microspacecraft, it is obviously desirable to minimize power consumption and flow rates. The results of the optical emission spectrometry show that the plasma source in the high-intensity mode absorbs the microwave power more effectively, expected to produce a high kinetic energy through a micronozzle if it is provided; furthermore, once the mode is raised up to the

high-intensity mode, the lower flow rate can sustain it at lower microwave powers. This means that the plasma source obtained here is likely to prove useful for the microthruster.

\section{Conclusions}

In the present work, an application of a microwave-sustained miniature plasma to an ultra small thruster has been presented, and both numerical and experimental investigations have been conducted. Numerical results show that the thruster provides a few $\mathrm{mN}$ levels of the thrust below the power of $10 \mathrm{~W}$, which could be enough for a station-keeping maneuver of microspacecraft. Optical 
emission spectrometry indicates that plasma discharges inside the quartz tube $1.5 \mathrm{~mm}$ in inner diameter using $4-\mathrm{GHz}$ microwaves involve a mode change depending on the absorbed power, and that lower flow rates can easily maintain the high-intensity mode at lower applied powers. These characteristics would be useful for the development of the microthruster. Further plasma diagnostics and developing a micronozzle will be the future work.

\section{References}

[1] K. Terashima, L. Howald, H. Haefke, H. Guntherodt, Thin Solid Films 281/282 (1996) 634.

[2] H. Yoshiki, Y. Horiike, Jpn. J. Appl. Phys. 40 (2001) L360.

[3] J. Hopwood, O. Minayeva, Y. Yin, J. Vac. Sci. Technol. B 18 (2000) 2446.

[4] A. M. Bilgic, U. Engel, E. Voges, M. Kuckelheim, J. A. C. Broekaert, Plasma Sources Sci. Technol. 9 (2000) 1.

[5] J. Mueller, in: M. M. Micci, A. D. Ketsdever (Eds.), Micropropulsion for Small Spacecraft, Progress in Astronautics and Aeronautics, vol. 187, American Institute of Aeronautics and Astronautics, Reston VA, 2000, p. 45.

[6] M. A. Lieberman, A. J. Lichtenberg, Principles of Plasma Discharges and Materials Processing, Wiley-Interscience Publication, New York, 1994, p. 306.

[7] H. Kousaka, K. Ono, Jpn. J. Appl. Phys. 41 (2002) 2199.

[8] H. Kousaka, K. Ono, Plasma Sources Sci. Technol. 12 (2003) 273.

[9] S. Kuchi-ishi, M. Nishida, Proceedings of the 26th International Electric Propulsion Conference, Kitakyushu, Japan, October 17-21, 1999, p. 193. 
[10] Y. Takao, K. Ono, K. Takahashi, Y. Setsuhara, Jpn. J. Appl. Phys. (to be submitted).

[11] J. D. Anderson, Jr., Computational Fluid Dynamics: the Basics with Applications, McGraw-Hill, New York, 1995, p. 456. 


\section{List of figures}

Figure 1. A schematic diagram of the miniature plasma thruster using microwaveexcited plasmas, which consists of a microplasma source and a micronozzle; the former yields the high thermal energy and the latter gives the kinetic energy.

Figure 2. Dependence of the electron density $n_{\mathrm{e}}$, the electron temperature $T_{\mathrm{e}}$, and the heavy particle temperature $T_{\mathrm{h}}$ (a); and of the thrust and the boundary layer thickness $\delta$ (b) on the absorbed power in plasmas $P_{\text {abs }}$ at the pressure of $100 \mathrm{kPa}$, the microwave frequency of $4 \mathrm{GHz}$, and the dielectric constant of 3.8.

Figure 3. A schematic diagram of the experimental setup and a cross-section view of the microplasma source (shown in the box).

Figure 4. Emission spectra and photographs of Ar plasma discharges at the incident power from the amplifier $P_{\text {in }}$ of $6 \mathrm{~W}$ (a) and $10 \mathrm{~W}$ (b). Microwave frequency $=4 \mathrm{GHz}$, argon flow rate $=100 \mathrm{sccm}$, feed pressure $=10 \mathrm{kPa}$, ambient pressure $=16 \mathrm{~Pa}$.

Figure 5. Dependence of emission intensities of Ar I line (811.5 nm) (a) and of reflective coefficients (b) on $P_{\text {in }}$. Other conditions are the same as in Fig. 4.

Figure 6. Dependence of emission intensities of Ar I line $(811.5 \mathrm{~nm})$ on $P_{\text {in }}$ for three argon flow rates: $100 \mathrm{sccm} ; 200 \mathrm{sccm}$; and $300 \mathrm{sccm}$. Corresponding feed pressures and ambient pressures: $10 \mathrm{kPa}, 16 \mathrm{~Pa} ; 15 \mathrm{kPa}, 23 \mathrm{~Pa}$; and $20 \mathrm{kPa}$, 29 Pa. Microwave frequency $=4 \mathrm{GHz}$. 


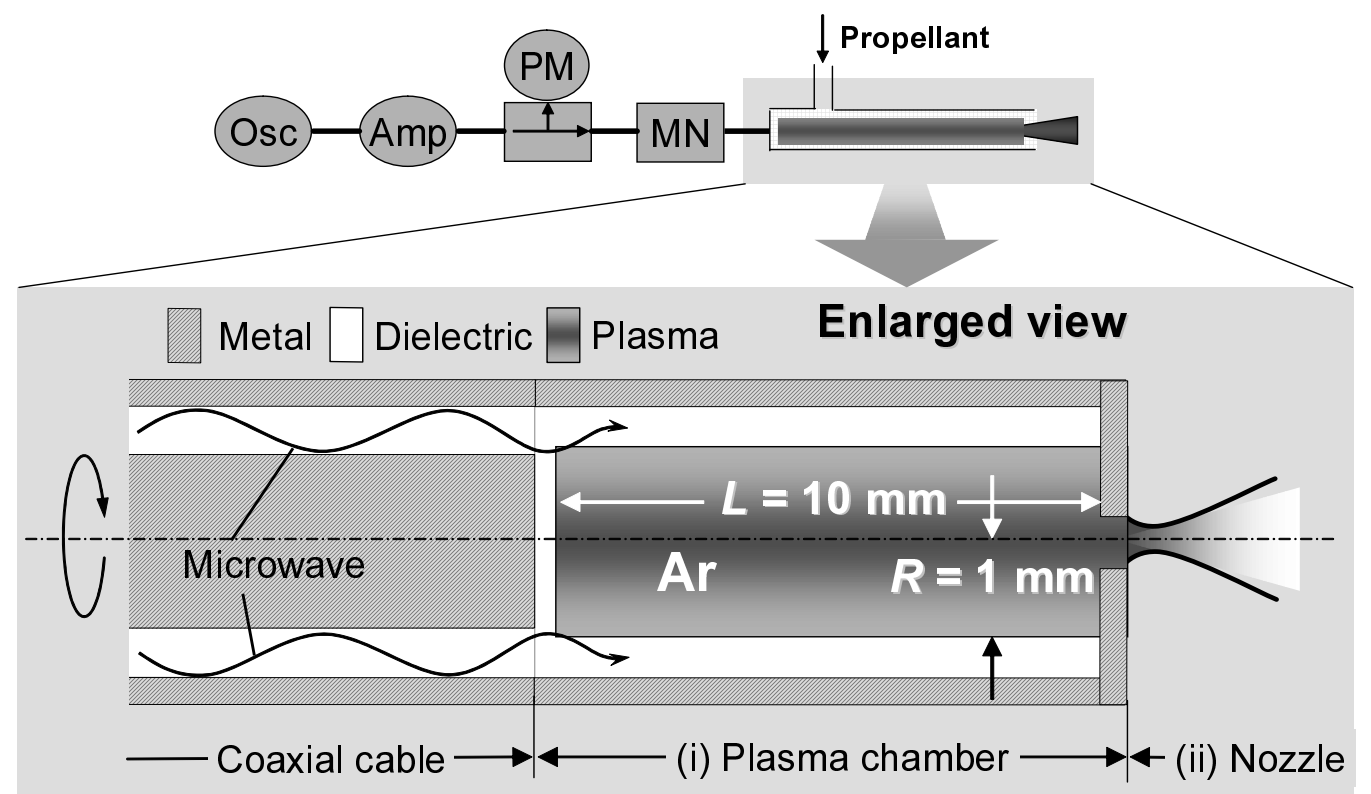

Y. Takao et al, Figure 1 

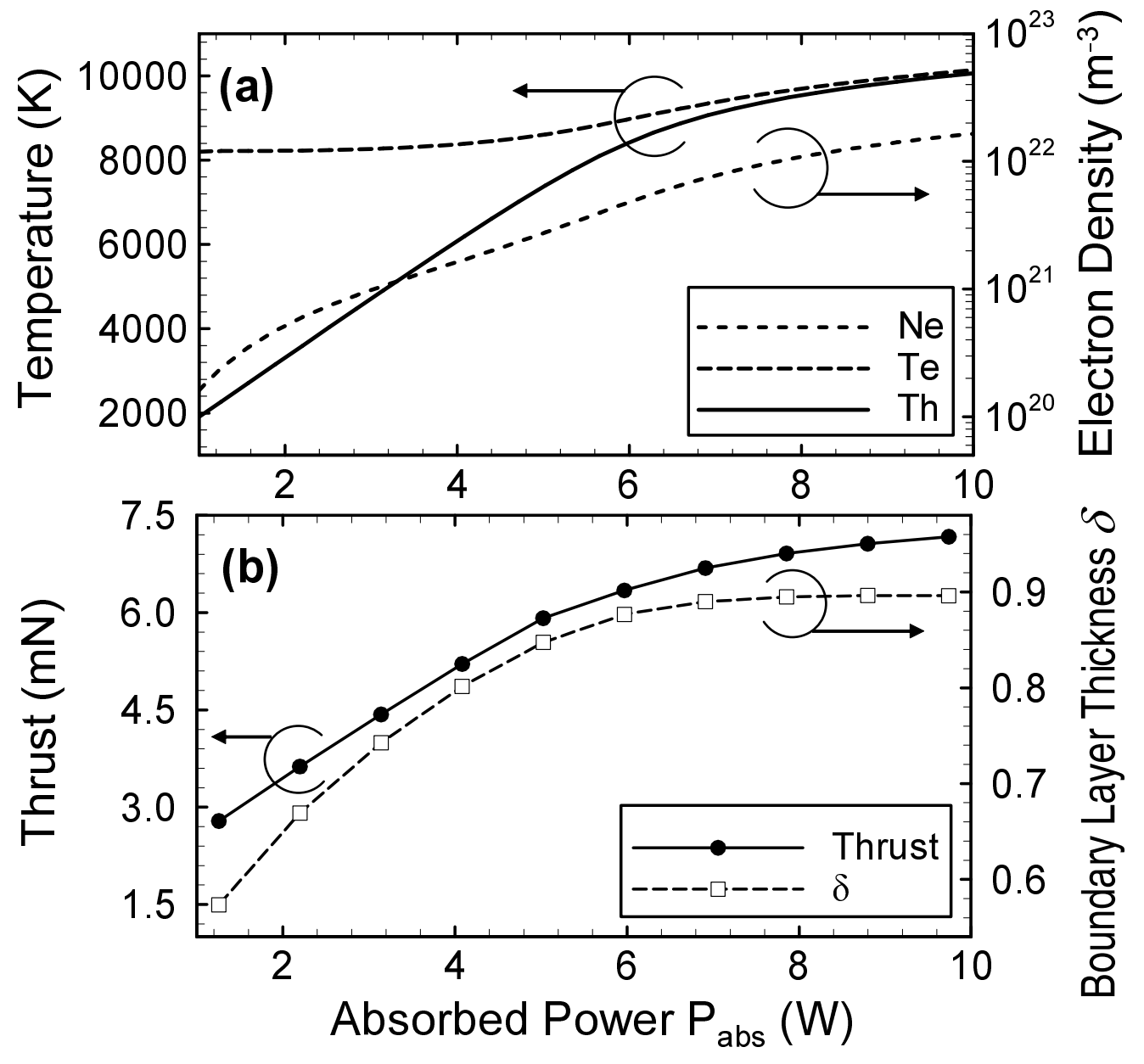

Y. Takao et al, Figure 2 


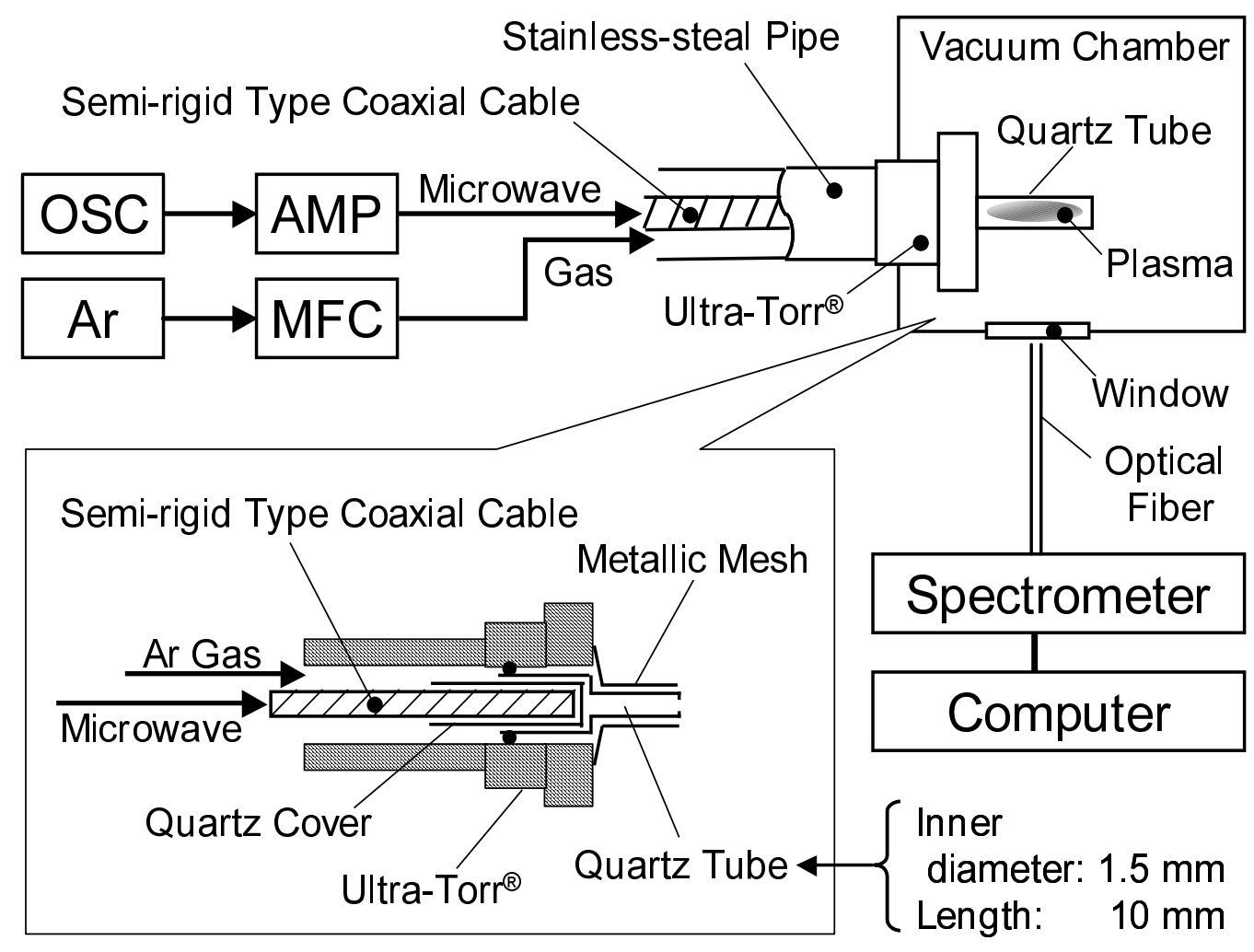

Y. Takao et al, Figure 3 

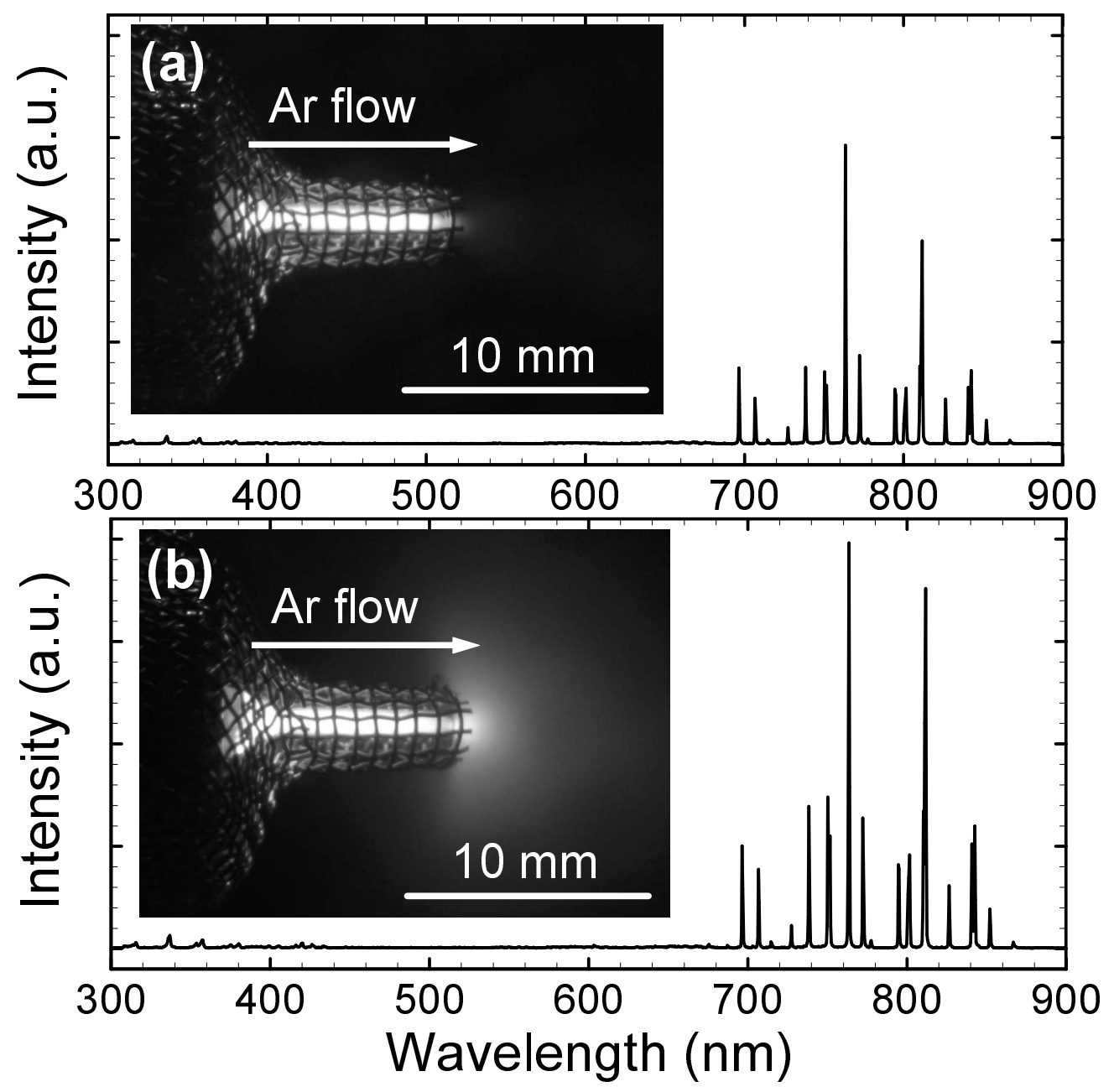

Y. Takao et al, Figure 4 


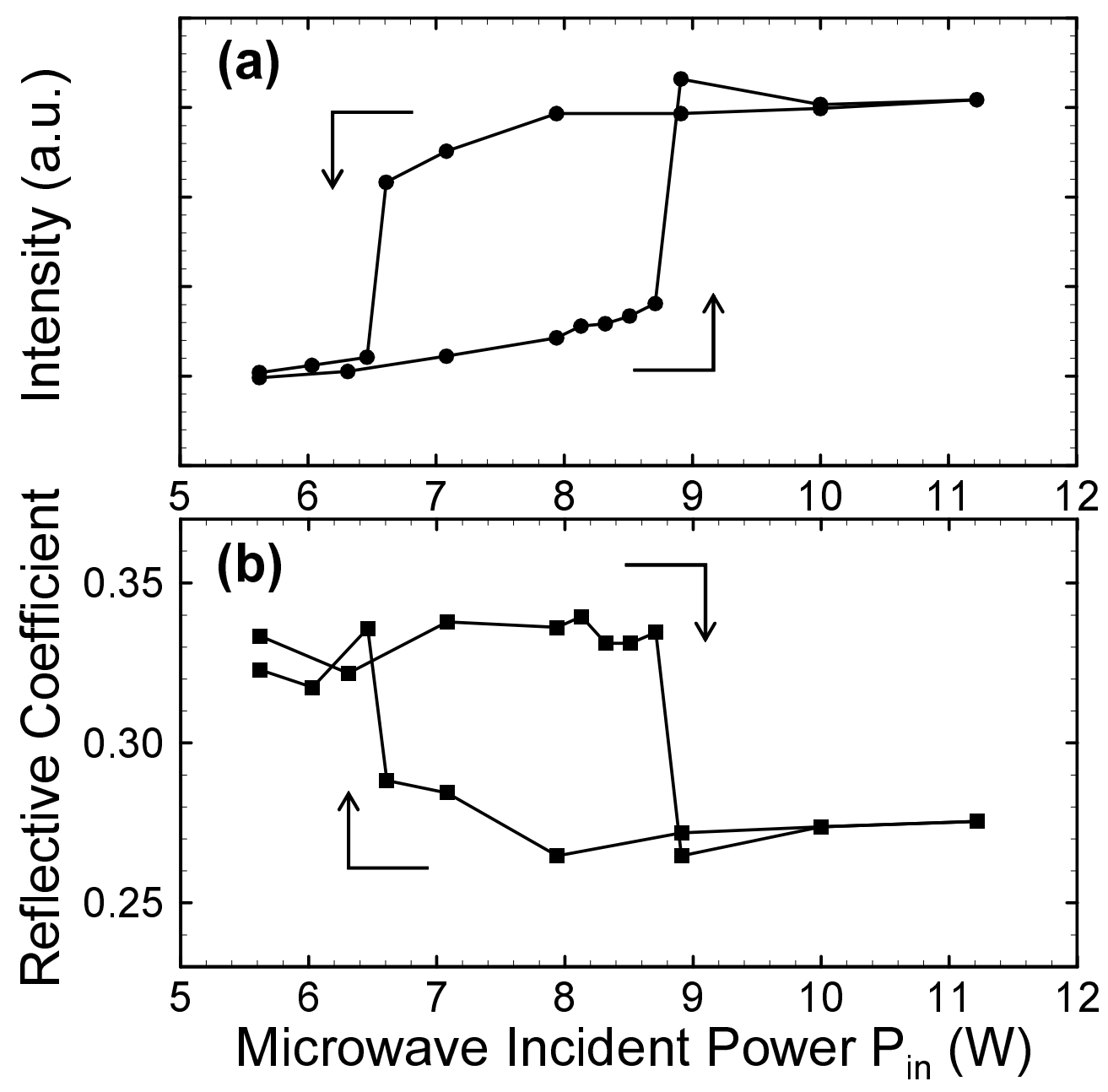

Y. Takao et al, Figure 5 


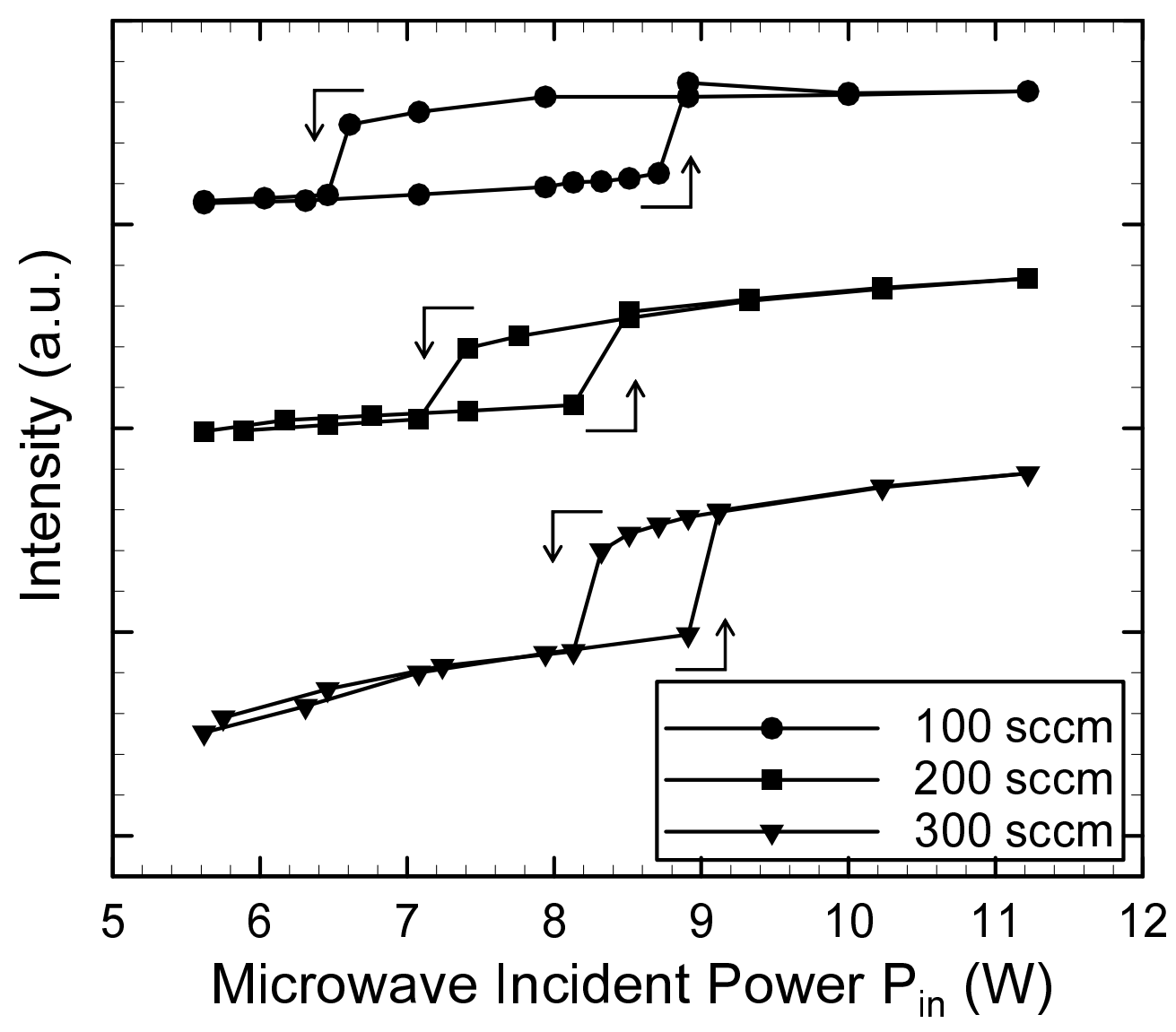

Y. Takao et al, Figure 6 